\title{
Wikileaks ante el derecho estadounidense
}

\author{
Wikileaks and the United States law
}

FELIPE CAMPOS ARLEGUI

Licenciado en Ciencias Jurídicas y Sociales, Universidad de Chile

\begin{abstract}
RESUMEN Este artículo expone las principales preguntas que a propósito del caso Wikileaks se ha hecho la doctrina estadounidense y cómo las ha respondido. Ello, en el intento de determinar cuál es el espacio para la libertad de expresión y de prensa que tutela el ordenamiento jurídico norteamericano y si los actos de Assange y Wikileaks serían o no reprochables.
\end{abstract}

PALABRAS CLAVE Wikileaks, libertad de expresión, libertad de prensa, Primera Enmienda.

ABSTRACT The current article exposes the main questions that the United States doctrine has faced and responded to, regarding the Wikileaks case. The purpose is to determine which is the room for freedom of speech and press that the North American legal system safeguards, and whether Assange's acts and Wikileaks would be reprehensible.

KEYWORDS Wikileaks, freedom of speech, freedom of press, First Amendment. 


\section{INTRODUCCIÓN}

La llamada "experiencia Wikileaks» ${ }^{1}$ y la consecuente publicación de documentos clasificados del gobierno estadounidense nos invitan a reflexionar acerca de cuál es la extensión de la libertad de expresión y de prensa en Estados Unidos en el contexto del rápido desarrollo y sencillo acceso a nuevas tecnologías de la información, las cuales han minimizado los costos y los tiempos de divulgación de diversos tipos de información que los gobiernos quisieran mantener en secreto.

Aportamos a la discusión las reflexiones de los principales docentes y profesionales estadounidenses expertos en libertad de expresión y en nuevas tecnologías, en lo que entendemos son las principales preguntas que debemos respondernos. Sobre todo aquella de si en el evento de ser juzgado en Estados Unidos, Assange y sus colaboradores deberían ser condenados o absueltos. ¿Sus actos están o no protegidos por la Primera

I. Por «experiencia Wikileaks» entendemos aquellas publicaciones de filtraciones realizadas por el sitio wiki fundado y dirigido por el activista Julian Assange y sus consecuencias mediáticas, políticas, diplomáticas y jurídicas. Sobre todo ponemos atención a las publicaciones más importantes, es decir, a las llevadas a cabo durante el año 20 Io:

- Video Collateral Murder del ejército estadounidense en que se registra desde dos helicópteros Apache cómo, mientras realizan labores de vigilancia en los suburbios de Bagdad, los soldados a cargo asesinan a civiles fríamente y sin ninguna justificación.

- Afghan War Diaries: material compuesto por 90.000 documentos de las Fuerzas Armadas de Estados Unidos. Entre ellos figuran informes de situación, informes sobre tiroteos y ataques aéreos, datos sobre incidentes sospechosos, etcétera, de los cuales se concluyen múltiples ataques a civiles con resultado de muerte, no investigados y archivados secretamente (Domscheit-Berg, 20II: I63).

- Irak War Logs: 391.832 documentos que cubrieron desde el I de enero de 2004 al 3 I de diciembre de 2009. Estaban constituidos por los informes que los soldados norteamericanos habían realizado dando cuenta de los incidentes que les habían parecido dignos de ser mencionados. En las redacciones se pueden leer todos los detalles: lugar exacto, día y hora, unidades militares participantes, cifra de muertos y si éstos eran norteamericanos, aliados, tropas iraquíes, insurgentes o civiles (O'Hagan, 20I 2: 244).

- Los telegramas diplomáticos: $25 \mathrm{I} .287$ cables escritos por 280 embajadas y consulados estadounidenses en I 80 países. De los documentos filtrados, un $40 \%$ estaban clasificados como confidenciales y un $6 \%$ eran secretos. No había ninguno de categoría alto secreto, pues ese material se omitía de la base de datos SIPRNET, además, y paradójicamente, las mejores historias procedían de aquellos con una confidencialidad más baja (Leigh y Harding, 20I I: 202-203). 
Enmienda estadounidense? ¿Se debe ser parte de la prensa tradicional para alegar la garantía a la libertad de publicar? ¿Se ha expuesto la seguridad nacional de Estados Unidos a un daño grave e irreparable?

Pensamos que las respuestas a estas preguntas — dada la innegable influencia de la democracia norteamericana en nuestros países, particularmente en temas de libertad de expresión e Internet- son clave para que tanto los medios de prensa tradicionales, las nuevas plataformas de comunicación y los ciudadanos reflexionemos sobre si, para defender nuestras democracias, podemos o no publicar información clasificada si ésta revela crímenes o delitos de los administradores del Estado sin exponernos a una condena. Al mismo tiempo, buscamos generar reflexión sobre hasta qué punto sería razonable utilizar el secreto y la seguridad nacional como argumentos que hagan reprochables tales publicaciones.

Nuestra tesis sostiene que una eventual condena a Assange se basaría en una errónea interpretación de la Primera Enmienda estadounidense.

\section{¿QUÉ PREGUNTAS SE HA HECHO LA DOCTRINA?}

\section{¿SE PODRÍA PROCESAR A JULIAN ASSANGE SEGÚN LA LEY DE FRAUDE INFORMÁTICO?}

Si bien esta ley a primera vista parece ser la más atingente para ser invocada, la doctrina la ha entendido aplicable sólo contra Bradley Manning ${ }^{2}$ (Benkler, 20II: 43), que al «excederse en el acceso autorizado» a las computadoras del gobierno, "comunica voluntariamente» información clasificada que «se podría utilizar para la lesión de los Estados Unidos, o en beneficio de cualquier nación extranjera» a «una persona no autorizada para recibirla».3

2. Exanalista del ejército de Estados Unidos. Fue detenido el 26 de mayo de 20 Io bajo la acusación de ser responsable de filtrar hacia Wikileaks, entre otros documentos, el video Collateral Murder (ver nota I). El 2I de agosto de $20{ }_{3} 3$ fue condenado a 35 años de prisión, ıo años más que el plazo en que los mismos documentos que él filtró serán desclasificados y habiéndose excluido el cargo de ayuda al enemigo. La justicia militar estadounidense dictó la sentencia con la sanción más extensa con que jamás se haya castigado una fuga de información, quedando pendiente el recurso de apelación.

3. The Computer Fraud and Abuse Act (CFAA) I 8 U.S.C. \$IO3० (a)(I). 
De hecho, en el único supuesto en que Assange podría ser procesado según esta ley, es en el supuesto de que haya incurrido en hacking 4 a los equipos del gobierno. Sabida la cooperación determinante de Bradley Manning en facilitar los documentos clasificados a Assange y las posibilidades que habría otorgado la red de servidores TOR, ${ }^{5}$ la alternativa parece alejarse. ${ }^{6}$

4. La conceptualización del término hacking ha variado a lo largo de las décadas permaneciendo hasta el día de hoy como un concepto con una cara positiva y otra negativa. Naturalmente, habiendo llegado este concepto a sede legislativa penal, esencialmente se ha referido a los «actos de acceso no autorizado a los sistemas de información, incluyendo las redes de computadores». Según se define en las leyes sobre delitos informáticos estatales y federales de los Estados Unidos, ello se extiende a las personas que extralimiten sus permisos autorizados dentro de una red informática determinada (Mcquade, 2009: 87).

5. The Onion Router (TOR) es un "proyecto cuyo objetivo principal es el desarrollo de una red de comunicaciones distribuida de baja latencia y superpuesta sobre Internet en la que el encaminamiento de los mensajes intercambiados entre los usuarios no revela su identidad, es decir, su dirección IP (anonimato a nivel de red) y que, además, mantiene la integridad y el secreto de la información que viaja por ella. Por este motivo se dice que esta tecnología pertenece a la llamada darknet o red oscura, también conocida con el nombre de deep web o web profunda. Para la consecución de estos objetivos se ha desarrollado un software libre específico. TOR propone el uso de encaminamiento de cebolla de forma que los mensajes viajen desde el origen al destino a través de una serie de routers especiales llamados «routers de cebolla» (en inglés onion routers). El sistema está diseñado con la flexibilidad necesaria para que pueda implementar mejoras, se despliegue en el mundo real y pueda resistir diferentes tipos de ataque. Sin embargo, tiene puntos débiles y no puede considerarse un sistema infalible». Disponible en <http:// es.wikipedia.org/wiki/Tor>.

6. En este punto debemos hacer referencia al sistema de protección de identidad de la fuente implementado en el sitio web de Wikileaks por el cual ni siquiera los administradores de éste podían acceder a identificar los sitios remitentes de información, por lo que tampoco cabría la posibilidad de complicidad o encubrimiento. Assange y su equipo han dicho que utilizan OpenSSL (un sistema de conexión segura de código abierto, como el utilizado por proveedores virtuales como Amazon), FreeNet (un método para compartir archivos peer-to-peer entre cientos o miles de ordenadores sin revelar su origen ni su propietario) y PGP (el sistema criptográfico de código abierto, abreviado de su jocoso nombre «Pretty Good Privacy») (Leigh y Harding, 20 I I: 67). Pero el principal dispositivo de protección de anonimato que utilizaron fue TOR. Domscheit-Berg, principal colaborador de Assange, explica cómo protegían a sus fuentes: «Nos encargábamos de que antes de llegar a nuestras manos, los documentos de informaciones explosivas dieran tantos 
Pero para el improbable escenario de comprobarse esa conducta, de todas formas la posibilidad de procesamiento y condena sería controvertida y altamente improbable, puesto que incluso existe el precedente de que la Corte Estatal de Kansas decretó la nulidad del concepto esencial de esta ley, esto es, del concepto de "acceso", fundamentalmente debido a su vaguedad. ${ }^{7}$ Por lo que en el caso de presentarse una acusación según esta ley ante la Corte Suprema podría inclusive aducirse la inconstitucionalidad de la misma o por lo menos de este concepto y hacer caer el requerimiento.

\section{¿SE PODRÍA PROCESAR A JULIAN ASSANGE SEGÚN LA LEY DE ESPIONAJE?}

Una opción que se ha sugerido es que la acusación seguramente se tramitaría bajo la Espionage Act. Sin embargo, la misma doctrina cuestiona que la Ley de Espionaje pueda ser utilizada con éxito contra Assange (Barron, 20II: 67-68).

\section{¿Podría Estados Unidos alegar que la Ley de Espionaje extiende su ámbito de aplicación a conductas fuera del país, realizadas por ciudadanos no estadounidenses, y que la protección de la Primera Enmienda no se les aplica?}

Acerca de su aplicabilidad en el extranjero, la Ley de Espionaje no da ninguna indicación expresa de que se pretenda aplicar extraterritorialmente, aunque los tribunales no han sido renuentes a aplicarla a la conducta de los estadounidenses en el extranjero. ${ }^{8}$ Pero ello no responde a la cuestión de si el acto está destinado a aplicarse fuera de los Estados Unidos a ciudadanos no estadounidenses, porque el espionaje es recono-

rodeos, pasaran por tantos procesos de cifrado y de eliminación de identidad como fuese posible, para que nadie lograra seguirles la pista. Ni siquiera nosotros mismos podíamos contactar con nuestras fuentes, por mucho que se tratara de algún asunto urgente. Nuestros remitentes no dejaban rastro alguno en la red, ni la menor huella dactilar, ni un solo byte que pudiera delatarlos» (Domscheit-Berg, 20I I: 45).

7. En State vs. Allen (1996) la Corte Suprema de Kansas sostuvo que su concepto era nulo debido a su vaguedad (Clough, 20I I: I 55 ).

8. En particular, porque el Congreso en I96I eliminó una disposición que limitaba su aplicación sólo «dentro del almirantazgo y jurisdicción marítima de los Estados Unidos y en alta mar, así como dentro de los Estados Unidos» (Elsea, 20I3: I6). 
cido como una forma de traición a la patria, que generalmente se aplica sólo a las personas que le deben lealtad a los Estados Unidos, pudiéndose suponer que el Congreso no lo considera como un delito que pueda ser cometido por un extranjero sin conexión con los Estados Unidos. Sin embargo, el único tribunal que parece haber abordado la cuestión concluyó lo contrario. Un juez de distrito en 1985 resolvió que un ciudadano de Alemania del Este podría ser procesado bajo las secciones 793 (b), 794 (a) y 794 (c). ${ }^{9}$

En ese caso, los acusados no estadounidenses que publiquen materiales que perjudiquen los intereses de Estados Unidos en formas que son legales en su jurisdicción, podrían ser procesados bajo la ley del país estando cubiertos por las garantías sustantivas de la Declaración de Derechos o Bill of Rights, por lo tanto la Primera Enmienda sí se les aplica. ${ }^{10}$

En una hipotética demanda contra The Guardian o Assange por la publicación de los cables diplomáticos, cabe señalar que hace más de cien años la Corte Suprema, en uno de los precedentes más importantes que extienden la protección constitucional más allá de las fronteras de Estados Unidos, declaró expresamente que «la libertad de expresión y de prensa» han sido de esos derechos tan «indispensables para un gobierno libre» que se aplican en el extranjero. ${ }^{11}$

En el caso Branzburg v. Hayes (I972), se dijo que no es probablemente un argumento plausible plantear que no se aplica la Primera Enmienda a la prensa extranjera. Por su parte, en United States $v$. I 8 Packages of Magazines (D.C. Cal. 1964), se estableció que:

9. United States vs. Zehe (I985). Por tener I) la información solicitada y obtenida ilegalmente sobre la defensa nacional de Estados Unidos; 2) entregó esa información a su propio gobierno; y 3 ) conspiró para hacerlo con la intención de que la información fuese utilizada en perjuicio de los Estados Unidos o en beneficio de la República Democrática Alemana, todos los cuales fueron delitos cometidos en Alemania del Este o en México (Elsea, 2013: г6).

Io. En esta construcción, es posible que los no ciudadanos que participan en la publicación de materiales revelados a ellos por otro estarán sujetos a enjuiciamiento si se puede demostrar que tomaron un papel activo en la obtención de la información. El caso no fue apelado. El acusado, Alfred Zehe, se declaró culpable en febrero de I985 y fue condenado a ocho años de prisión, pero fue negociado como parte de un «intercambio de espías» con Alemania del Este en junio de ese año (Elsea, 20I3: I7).

I I. Downes vs. Bidwell, I82 U.S. 244, 282-83 (I90I) (Benkler, 20II: 36). 
La Primera Enmienda protege a la población de este país... La Primera Enmienda con seguridad fue diseñada para proteger los derechos de los lectores y distribuidores de publicaciones no menos que los de los escritores o impresores. De hecho, la esencia del derecho a la libertad de prensa de la Primera Enmienda no es tanto el derecho de imprimir, sino que el derecho a leer. Los derechos de los lectores no deben ser restringidos debido a la procedencia geográfica de los materiales impresos (Elsea, 20I3: 22).

\section{¿El gobierno de Estados Unidos podría demostrar el requisito de la intención especifica para lograr su condena?}

En los próximos apartados utilizaremos las opiniones de los más destacados profesores de derecho y de abogados expertos en la materia, en específico de Jerome A. Barron, profesor de la Universidad George Washington; Yohai Benkler, profesor de la Universidad de Harvard; Shaina Jones y Jay Ward Brown, abogados especialistas en medios; Jennifer K. Elsea, abogada del Servicio de Investigación del Congreso de Estados Unidos; Gabriel Schoenfeld, miembro del Instituto Hudson en Washington y del Instituto Witherspoon en Princeton, y Geoffrey Stone, profesor de la Universidad de Chicago.

a) Posición del profesor Barron. Primero que todo se refiere a la constitucionalidad de la ley, plantea que es un estatuto que tiene casi cien años y que, según los actuales estándares de la Primera Enmienda, el lenguaje de muchas de sus disposiciones es vago y demasiado amplio.

Luego, refiriéndose propiamente al punto en cuestión, piensa que el caso AIPAC es ilustrativo, dado que en éste el juez Ellis se negó a desestimar la acusación según la Ley de Espionaje (Barron, 20 I I: 68), sin embargo al mismo tiempo declaró que un enjuiciamiento exitoso tenía que cumplir con el requisito de probar la intención específica. «La información debe estar relacionada con la defensa nacional, ya sea tangible o intangible, debiendo ser necesariamente aquella cuya divulgación sea potencialmente perjudicial para los Estados Unidos, y el acusado debe saberlo» (Barron, 20II: 69).

Por otra parte, el profesor Barron recuerda que antes de que se publicaran los cables diplomáticos, Assange se dirigió al Departamento de Estado preguntando acerca de los criterios que podrían enmarcar un actuar 
correcto o incorrecto de su parte desde el punto de vista estadounidense (Barron, 20I I: 69). El Departamento de Estado se negó a cooperar. Por lo tanto, dice, puede ser difícil demostrar que existió una deliberada intención de dañar a Estados Unidos. Uno podría esperar que su abogado defensor argumente que sus acciones demuestran lo contrario.

Según su postura, incluso si se cumple con el requisito específico de la intención de la Ley de Espionaje, la persecución contra Assange por publicar a través de Wikileaks aún tendría que sobrevivir a la cuestión de la Primera Enmienda (Barron, 20 I I: 69).

b) Reflexión de Jones y Ward. Según ellos, parece claro que Assange divulgó documentos relacionados con la defensa nacional a personas que no tenían derecho a recibirlas, que al menos algunos de estos documentos eran secretos, que objetivamente su divulgación tendría el potencial de dañar la seguridad de los Estados Unidos, y que Assange era consciente del carácter secreto de los documentos. Si esto es correcto, y si el juez al que se le asignase el caso adhiere a la articulación de la carga del gobierno razonada por el juez Ellis, entonces la única pregunta que queda es si el gobierno puede demostrar que Assange sabía que la divulgación de los documentos era ilegal, y no obstante ello procedió, es decir, que actuó con mala intención para desobedecer la ley (Jones, 2OII: I35).

Piensan que si uno de los medios de comunicación dominantes en Estados Unidos hubiese sido el que dio a conocer los mismos documentos que Wikileaks, respondería esta acusación declarando que su intención era la de informar al público sobre asuntos pertinentes al self-government, y que no se violó ni la ley ni se tuvo la intención de dañar al gobierno. Así se ha interpretado la intención por los tribunales en circunstancias análogas. ${ }^{12}$

I2. Por ejemplo, para condenar por imposición intencional de angustia emocional se requiere probar que el acusado actuó intencionalmente o por imprudencia y que las consecuencias naturales y probables de la acción podrían causar la angustia emocional del demandante. Las cortes han determinado que la publicación de lleno intencional de un informe de prensa no constituye una intención de infligir daño. Por ejemplo, Levin v McPhee, 9I7 F. Supp. 230 (SDNY I996), I I9 F. 3 d I 89 aff'd (2d Cir I997.); Witherspoon v Phillip Morris Inc., 964 F. Supp. 455, 463 (DDC 1997) (que sostiene que la intención de causar daño debe ser dirigida específicamente hacia la persona que se queja de tal daño, en lugar de hacia el público en general) (Jones, 20II: I35-I36). 
Ahora, refiriéndose específicamente a la liberación de los cables diplomáticos y la prueba de la intención específica, citan a Baruch Weiss, abogado de la defensa en el caso AIPAC, quien señaló que «mucho antes de la publicación de los cables, [Assange] escribió una carta al gobierno de Estados Unidos, la que fue entregada a nuestro embajador en Londres, pidiendo sugerencias para sus redacciones. El Departamento de Estado se negó. Assange después escribió otra carta, reiterando que «Wikileaks no tiene absolutamente ningún deseo de poner a las personas individuales en riesgo significativo de daño, ni queremos dañar la seguridad nacional de los Estados Unidos» (Jones, 20I I: I36).

c) Opinión de Jeniffer K. Elsea, abogada del Servicio de Investigación del Congreso. Piensa que existe una amplia autoridad legal para procesar a las personas que provocan la difusión o difunden los documentos en cuestión, siempre y cuando el elemento de intención pueda ser probado y el daño potencial a la seguridad nacional pueda ser demostrado. Agrega que, sin embargo, hay una posición interpretativa de la sección 793, que prohíbe la comunicación, transmisión o entrega de información protegida a cualquier persona que no tenga derecho a tomar posesión de ella, que excluye la publicación del material (Elsea, 20I3: I5).

Por otra parte, nos dice que el estatuto descrito en el apartado anterior se ha utilizado casi exclusivamente para procesar a personas con acceso a la información clasificada (y la correspondiente obligación de protegerla) que la pusieron a disposición de agentes extranjeros o agentes extranjeros que obtuvieron la información clasificada ilegalmente mientras se encontraban en Estados Unidos. Argumenta que el Servicio de Investigación del Congreso no tiene conocimiento de algún caso en que un editor de la información obtenida a través de la divulgación no autorizada hecha por un empleado del gobierno haya sido procesado por publicarla. Es posible que las implicaciones relacionadas con la Primera Enmienda hagan difícil tal acusación, y eso sin hacer mención de las contingencias políticas que sobrevendrían basadas en preocupaciones sobre la posible intención de censura por parte del gobierno (Elsea, 2013: I6).

El gobierno tendría la carga de probar la «mala intención» de Wikileaks o Assange, lo que además de ser de suyo complejo, ha sido percibido como un obstáculo para el enjuiciamiento de los periodistas en el pasado (Elsea, 20I3: I6). 


\section{¿LAS PUBLICACIONES DE ASSANGE Y WIKILEAKS ESTÁN PROTEGIDAS POR LA PRIMERA ENMIENDA?}

Más allá de la exigencia de que el gobierno demuestre la intención necesaria para asegurar una condena en virtud de la Ley de Espionaje, situación que, como hemos visto, se observa de muy compleja consecución, quedaría, sin embargo, la cuestión de si su aplicación a Wikileaks sería constitucional. Como se ha señalado, los tribunales nunca se han enfrentado directamente con el procesamiento de un órgano de prensa o a un periodista individual bajo la Ley de Espionaje, al menos no en la era de la moderna jurisprudencia de la Primera Enmienda (Jones, 20I I: I40).

\section{¿Wikileaks es parte de la prensa?}

a) Posición del profesor Barron. Apunta a que hoy algunos blogeros reciben pases de prensa y participan en las conferencias de prensa presidenciales (Barron, 20I I: 70). Dice que el sitio web Wikileaks existe con el fin de distribuir información, pero que sin embargo algunos sostienen que Assange no es un periodista (Barron, 20I I: 70). También agrega el argumento que pretende excluir a Wikileaks de la protección conferida por la garantía de la libertad de prensa debido a que su contenido se transmite a través de la Internet en lugar de las páginas impresas de los diarios. Este argumento, dice, es especialmente débil en este caso, en el que los medios de comunicación tradicionales han trabajado muy cerca de Wikileaks (Barron, 20I I: 70). La cooperación de estos medios tradicionales ha servido para aumentar el alcance de sus publicaciones.

Plantea que las publicaciones demuestran una interacción entre Wikileaks y la prensa establecida. Cuando los primeros envíos de material sobre la guerra de Afganistán fueron publicados por Wikileaks, la reacción de la prensa tradicional fue de crítica. Los nombres de soldados y otros miembros habían sido revelados y la prensa informó de personas cuya vida se habría puesto en peligro (Barron, 20II: 70).

Luego, dice que al momento de publicarse los archivos relativos a Irak y versiones posteriores de los cables diplomáticos, Wikileaks editó el material y que la prensa tradicional lo elogió, pues tuvo acceso previo al material que se publicaría, por lo que en lugar de competir, Wikileaks aparentemente había decidido cooperar con la prensa establecida, 
y que, de hecho, Wikileaks se había convertido en una especie de prensa adjunta.

Argumenta que, en casos como éste, para él se debe aplicar un «examen riguroso", pues un interés gubernamental apremiante podría llegar a prevalecer sobre las consideraciones de la Primera Enmienda (Barron, 2OII: 7I).

Si Assange es procesado, estima que lo más probable es que alegue que el sitio web Wikileaks es parte de la prensa. Cita a Baruch Weiss que señaló que ni el Washington Post ni The New York Times están siendo investigados por publicar el mismo material que Julian Assange publicó en Wikileaks (Barron, 20II: 68). La razón de esto sería clara: la garantía de la libertad de prensa. Weiss dice que la práctica del Departamento de Justicia ha sido la de «abstenerse de introducir acusaciones sobre las fugas contra los medios de comunicación tradicionales» (Barron, 20I I: 68). Sin embargo, Julian Assange está siendo investigado. Weiss sugiere que "[el procurador general] Holder puede sentirse animado a dirigirse contra Wikileaks, [sólo] porque no tiene el aspecto o la impresión de tratarse de un medio de comunicación tradicional» (Barron, 20I I: 68).

b) Reflexión del profesor Benkler. Para Benkler la diferencia más obvia entre Wikileaks y los medios de comunicación tradicionales es la identidad de la organización. Estos últimos son familiares y tenemos una suposición general sobre su forma de organización: se preocupan por obtener los datos correctos y de ser «responsables», dice, en la presentación de las noticias. Expresa que, entonces, la línea divisoria importante es entre los medios y los periodistas establecidos, por un lado, y la cultura descentralizada, informal o cuasi-formal de expresión en Internet. Qué podría explicar esta diferencia, se pregunta. La intuición, dice, probablemente tomaría una forma análoga a la expresión formulada por Jonathan Klein, quien, poco antes de asumir como presidente de CNN dijo que «no podría haber un contraste más marcado entre las múltiples capas de pesos y contrapesos (de un medio de prensa) y un chico sentado en su sala de estar en pijama escribiendo lo que piensa", haciendo referencia a los blogeros que habían exhibido un informe de 60 minutos de la historia militar del presidente George Bush. Klein ya no conduce $\mathrm{CNN}$, pero el tratamiento desdeñoso hacia la blogósfera desde los medios tradicionales no ha desaparecido (Benkler, 20I I: 37). 
Benkler recuerda que la cobertura de Wikileaks del propio The New York Times se combina con retratos poco halagadores de Julian Assange. ${ }^{13}$ Piensa que estas descripciones parecen representar una profunda ansiedad y crisis de identidad de los medios tradicionales, tal vez exhibiendo un miedo existencial a que los días de gloria de su profesión hayan pasado, tal vez sencillamente por envidia por el hecho de que la mayor primicia de 2010 fue generada por alguien que no era miembro del club. Cualquiera que sea la razón de este retrato poco favorecedor, dice, no puede constituir la base de un principio constitucional (Benkler, 2OII: 38 ).

Plantea, citando a la Corte Suprema, que «la libertad de prensa es un derecho tanto del panfletario solitario que utiliza papel carbón o un mimeógrafo, como de la gran editorial metropolitana que utiliza los últimos métodos de fotocomposición». ${ }^{14}$

La organización de «frenos y contrapesos» institucional-procesales de los que Klein habló, dice, no pueden sostener una distinción que haga la diferencia constitucional.

La diferencia entre medios de Internet y los medios de comunicación tradicionales no puede, entonces, ser el tamaño o la complejidad organizativa. Funcionalmente, reflexiona, es más importante proporcionar protección constitucional sólida a los miembros más débiles de Internet, que tienen menos visibilidad pública y recursos para resistir la presión de los funcionarios del gobierno, que hacer hincapié en los derechos de los miembros de un ente organizativa y económicamente más fuerte. ${ }^{15}$

I3. A quien el periódico describió variadamente como «hombre perseguido», que «se registra en hoteles bajo nombres falsos, se tiñe el pelo, duerme en los sofás y pisos, y usa efectivo en lugar de tarjetas de crédito, a menudo prestado de amigos», o que usaba una "camisa blanca sucia, zapatillas deportivas y calcetines blancos sucios que se derrumban alrededor de sus tobillos. Olía como si no se hubiera bañado en días».

I4. Benkler (20II: 38 ) citando Branzburg v. Hayes, 408 U.S. 665, 704 (I972).

I 5. "Cuando el senador Bunning y el representante Kyl llaman a la revelación de The New York Times del programa de espionaje interno NSA 'traición a la patria', había poco riesgo de éxito de un proceso penal contra The New York Times, o que su editor se llegara a encontrar bajo arresto domiciliario, que llevase un brazalete en el tobillo. El enorme poder económico, social y cultural del New York Times implica que las garantías constitucionales no tendrían que 'patear' para evitar tal eventualidad. Lo mismo no es necesariamente cierto para un hombre a quien el Vicepresidente de Estados Unidos des- 
Aunque el director ejecutivo de The New York Times haga hincapié en que ve a Assange como «una fuente, enfáticamente no un socio, y no realmente un periodista», esta negación sentenciosa de la membresía en el club no hace una diferencia constitucional (Benkler, 20I I: 39).

Cita el caso Von Bulow vs. Von Bulow (2nd Cir. 1987), en que la Corte de Apelaciones concluyó subrayando que la membresía en el club de periodistas establecidos no es necesaria para la protección constitucional. ${ }^{16}$

El elemento definitorio para Benkler, entonces, sería la intención al momento de la recolección y su función, no el modo de difusión: la intención de recolectar información para su difusión pública. Para el profesor Benkler, simplemente, no puede haber ni la más remota duda que todo el propósito de Wikileaks es la recopilación de información para la difusión pública y el uso de los medios de comunicación tradicionales como la vía principal, destacando este hecho, aunque no es constitutivo o un elemento necesario de la defensa. El interés de que se trata no es individual, argumenta, sino que sistémico. Es: «El interés de la sociedad en la protección de la integridad del proceso de recopilación de noticias, para garantizar la libre circulación de la información al público» (Benkler, 20I I: 39-40). Tampoco para él es relevante si su interés es o no perjudicar al gobierno de Estados Unidos. ${ }^{17}$

cribe como un 'terrorista de alta tecnología', y que The New York Times describe como 'un hombre perseguido', mientras que su director ejecutivo hace hincapié en que lo ve como 'una fuente', enfáticamente no un socio, y no realmente un periodista» (Benkler, 2OII: 38$)$.

I6. «Aunque la experiencia previa como periodista profesional puede ser una evidencia persuasiva de la intención presente de reunir [documentación clasificada] para los efectos de difusión, no es condición sine qua non. La carga de hecho puede ser sostenida por alguien que es un novato en el campo. Además, la protección puede ser solicitada por alguien a quien no se asocia tradicionalmente con la prensa institucionalizada porque la función informativa afirmada por los representantes de la prensa organizada [...] También se lleva a cabo por los profesores, encuestadores políticos, novelistas, investigadores, académicos y dramaturgos» (Benkler, 20I I: 39-40, citando 274 U.S. 357 (I927)).

I7. «El propósito de la protección de la prensa es sistémico y funcional: servir a un público más ilustrado, que es condición previa para que una democracia funcione bien. La motivación que impulsó a cualquier individuo dado para avanzar en ese objetivo es totalmente irrelevante para la cuestión central. Un periodista no se mide por el hecho de que investiga y publica con el fin de servir a la democracia, engrandecer su nombre 
Llega, entonces, a la conclusión de que, como cuestión de doctrina de la Primera Enmienda, Wikileaks tiene derecho a la protección disponible para una amplia gama de miembros del cuarto poder (Benkler, 20I I: 4I).

Sostiene que la esfera pública en red se desarrolla como un conjunto de diversos actores, desde blogeros individuales, pasando por organizaciones no lucrativas como la Fundación Sunlight, ${ }^{18}$ pequeñas publicaciones en línea comerciales y grandes grupos descentralizados de activistas políticos como Daily $\operatorname{Kos}^{19}$ o Townhall, ${ }^{20}$ que vienen a jugar un papel cada vez más importante en la construcción de la esfera pública, siendo de importancia funcional el divorciarse de la protección constitucional que se entendía en la medida en que el solicitante formaba parte del modelo del siglo XX de los grandes medios de comunicación (Benkler, 20I I: 42).

Para él, no es sostenible dar a Wikileaks y a Assange un tratamiento diferente que al The New York Times y sus periodistas con fines de censurar previamente o de modo ex post procesar penalmente. Ello, en consonancia con su interpretación de la Primera Enmienda y la protec-

o hacer dinero. Fox News no sería menos merecedor de la libertad de prensa si se encontraran una serie de memorandos internos que revelen que su primer motivo era el de socavar la capacidad del presidente Obama para gobernar, en lugar de informar al público. Indagar en las motivaciones políticas o personales de los oradores abre la puerta a la forma más perniciosa de la censura: la definición de algunas motivaciones políticas como bases legítimas para el habla, y otras como ilegítimas no son elegibles para la protección. Un reportero que opera por convicción política es tan digno de protección como un reportero que busca hacer dinero, convertirse en una celebridad, o humildemente servir al interés público» (Benkler, 20II: 4I).

I 8. La Sunlight Foundation es una organización no partidista sin fines de lucro. Fundada en 2006, su objetivo es el de aumentar la transparencia y la rendición de cuentas tanto del Congreso de los Estados Unidos, del poder Ejecutivo, como de los gobiernos estatales y locales. Disponible en <http://en.wikipedia.org/wiki/Sunlight_Foundation>. Traducción del autor.

19. Es un blog político estadounidense que publica noticias y opiniones desde un punto de vista liberal. Funciona como un foro de discusión y blog colectivo para activistas cuyos esfuerzos se dirigen principalmente hacia influir y fortalecer el Partido Demócrata con un enfoque particular en las políticas y los candidatos progresistas. Disponible en $<$ http://en.wikipedia.org/wiki/Daily_Kos>. Traducción del autor.

20. Es una publicación web y una revista impresa que publica noticias y opiniones desde un punto de vista conservador. Sus columnistas a menudo aparecen como comentaristas invitados en C-SPAN, MSNBC, CNN y Fox News Channel. Disponible en <http:// en.wikipedia.org/wiki/Townhall>. Traducción del autor. 
ción de la libertad de prensa. Según él, un enjuiciamiento a Wikileaks o a Assange seguramente no prosperará bajo la actual doctrina de la Primera Enmienda. Añade que en el improbable caso de que la persecución tenga éxito, sólo lo haría a costa de empeorar la Primera Enmienda desde la perspectiva de la libertad de prensa en la era de la Internet (Benkler, 2OII: 3-4).

c) Posición de Jones y Ward. Con respecto a la versión de abril de 2010 del video Asesinato colateral, ${ }^{21}$ Jones y Ward plantean que sería difícil argumentar que Wikileaks no realiza ninguna función periodística tradicional: no sólo edita y titula el video, sino que, al parecer, envió personal a Irak para entrevistar a los testigos iraquíes sobre el incidente para asegurar la veracidad de la información, para luego publicarlo. Algo que Reuters había intentado sin éxito durante años, fue un hecho ampliamente cubierto por organizaciones de noticias en Estados Unidos $y$ en el extranjero (Jones, 20II: I37).

Plantean que es difícil discernir un argumento convincente de que cualquiera de las diferencias fácticas entre Wikileaks y las «organizaciones típicas de noticias» importe cuando se trata de buscar refugio bajo la Primera Enmienda. Correctamente entendida, argumentan, la Primera Enmienda «no se preocupa si Wikileaks es un acreditado distribuidor de noticias, un grupo de hackers 'de sombrero negro' o un circo ambulante», precisamente porque la protección que ésta ofrece «depende de lo que Wikileaks publica, no de lo que es».22 De hecho, es bien aceptado, dicen, que «la prensa en su connotación histórica comprende todo tipo de publicación que ofrece un vehículo de información y de opinión». ${ }^{23}$

Se argumenta que los tribunales nunca se han enfrentado directamente con el procesamiento de un órgano de prensa o de un periodista individual bajo la Ley de Espionaje, al menos no en la era de la moderna jurisprudencia de la Primera Enmienda (Jones, 20 I I: I39).

\footnotetext{
2I. Disponible en <http://www.collateralmurder.com/ $>$.

22. Jones (20II: I39) citando a su vez a Andy Greenberg, Is WikiLeaks a Media Organization? The First Amendment Doesn't Care, Forbes, disponible en <http://www. forbes.com/sites/andygreenberg/20 I I/04/2 I/is-wikileaks-a-media-organization-the-firstamendment-doesnt-care/>.

23. Jones (20II: I39) citando a su vez Lovell vs. City of Griffin, 303 U.S. 444, $45^{2}$ (1938).
} 
En una serie de casos que comienzan con Smith vs. Daily Mail en I979, la Corte Suprema en varias ocasiones ha subrayado que «la información veraz sobre un asunto de interés público» recibe muy amplia protección ante la responsabilidad penal o civil. ${ }^{24}$

Conocido como «el principio Daily Mail», esta exigencia constitucional de base fue explicada recientemente por la Corte Suprema en Bartnicki vs. Vopper ${ }^{25}$ un caso que surgió bajo la ley federal de escuchas telefónicas ${ }^{26}$ que tipifica como delito y crea una acción civil para la interceptación ilegal y la difusión de comunicaciones por cable o inalámbricas (Jones, 20II: I40). Describen que cuando la discusión se fundamenta sobre información veraz acerca de un asunto de importancia pública, tal cual reafirmó la Corte, «los funcionarios del Estado no pueden castigar constitucionalmente la publicación de la información, en ausencia de una necesidad... de primer orden» (Jones, 20II: I4I).

Cabe destacar que esta protección dejaría de operar, dijo la Corte, cuando el editor va más allá de saber que acepta material obtenido ilegalmente por otro, llegando a la participación real en la obtención ilegal del material, lo que constituiría hacking en su matiz descrito como delito penal (Jones, 20I I: I4I).

Reflexionan que tal vez la analogía más cercana a Wikileaks en estos aspectos es presentada por Jean v. Mass. State Police en que se aplicó el precedente Bartnicki. ${ }^{27}$ En este caso, Mary Jean publicó en Internet una grabación de audio y video de un arresto y allanamiento residencial sin orden judicial, a pesar de su conocimiento de que la grabación se había hecho ilegalmente.

Suponiendo que en el caso de Assange éste hubiese actuado conjuntamente con sus fuentes desde los actos iniciales presuntamente ilegales de adquisición y divulgación, viciando la aplicación del principio Daily

24. Jones (20I I: I39) citando a Smith v. Daily Mail, 443 U.S. 97, I03 (I979), en el contexto de la afirmación de que un periódico publicó el nombre del agresor de menores, sostiene que «si un periódico obtiene legalmente información veraz acerca de un asunto de importancia pública, luego los funcionarios estatales no podrán sancionar constitucionalmente la publicación de la información, en ausencia de la necesidad de promover un interés estatal de primer orden».

25. 532 U.S. 5 I 4 (200I).

26. Wiretapping Act.

27. Jones (20II: I4I) citando 492 F.3d 24 (Ist Cir. 2007). 
Mail en primera instancia, y suponiendo que el gobierno podría cumplir en lo demás con la carga de la prueba, ¿el gobierno tendría éxito en demostrar que la aplicación de la Ley de Espionaje a Assange sirve legítimamente a un interés de "primer orden»? La protección de la seguridad nacional, incluidos los secretos necesarios para la seguridad, parecen claramente calificar como un interés nacional de primer orden (Jones, 20II: I4I). La pregunta más precisa sería si el contenido de los documentos publicados por Wikileaks reflejan esos intereses. Por ejemplo, un comentarista ha argumentado que si los cables revelan los nombres de agentes secretos en Afganistán o Irak, o se refieren a planes de guerra futuros o contienen algo referido a prácticas similares, el estándar de la norma podría superarse. ${ }^{28}$

d) Opinión del abogado Elsea del Servicio de Investigación del Congreso. Elsea opina que el hecho de que Wikileaks no sea una organización de recopilación de noticias y de publicaciones tradicional probablemente hará poca diferencia en el análisis de la Primera Enmienda. Reflexiona que el Tribunal Supremo no ha establecido límites claros entre la protección de la expresión y de la prensa, ni ha tratado de desarrollar criterios para determinar lo que constituye «la prensa», para poder calificar a sus miembros con privilegios que no estén disponibles para cualquier persona. ${ }^{29}$

\section{¿Quién es el garante de que los secretos del gobierno permanezcan como tales?}

Mucho optimismo se generó en materia de expansión de la libertad de prensa en 2008, cuando el candidato presidencial Barack Obama prometió aumentar la transparencia y reducir los secretos gubernamentales. Sin embargo, convertido en presidente, Obama ha seguido una tendencia hacia un mayor secretismo del gobierno, incluso en comparación con

28. Jones (20II: I4I) citando a su vez a Andy Sellars, WikiLeaks and the First Amendment, Andy on the road, disponible en <http://andyontheroad.wordpress. $\mathrm{com} / 20 \mathrm{IO} / \mathrm{I} 2 / \mathrm{I}$ I/wikileaks-and-the-first-amendment/>.

29. Elsea (2013: 22), recomienda ver también Congressional Research Service, The Constitution of the United States: Analysis and Interpretation, Sen. Doc. I08-17, at I083-86 (2002), disponible en <http://crs.gov/conan/default.aspx?mode=topic\&doc= Amendmentor $\cdot x m l \& t=\left.2\right|_{3}>$. 
gobiernos anteriores. De acuerdo con la Oficina de Supervisión de Seguridad de la Información, la agencia federal que supervisa los sistemas de clasificación del gobierno, el costo anual de la clasificación se ha elevado a más de ı०,7 mil millones de dólares, sobrepasando el umbral de ıо mil millones por primera vez, porque muchas de las decisiones gubernamentales que solían ser públicas están siendo clasificadas como secretos (American Academy, 20I2: 20). En 20I0, aproximadamente 224.000 documentos fueron clasificados: un aumento del $22 \%$ respecto a 2009 (American Academy, 20I 2: 20).

Las solicitudes motivadas por la Ley de Libertad de Información también han aumentado en respuesta al creciente secretismo gubernamental. Hubo más de medio millón de solicitudes en 2010, 40.000 más que en 2009, y la burocracia del gobierno respondió a I 2.400 solicitudes menos que en el año anterior. Más solicitudes se están haciendo y menos están siendo procesadas (American Academy, 20I 2: 20).

Pero hay algo peor: el gobierno de Obama ha invocado el secreto de Estado más que cualquier otro en los tiempos modernos, incluyendo la administración del presidente Bush, en lugar de responder a las solicitudes de información clasificada. El presidente Obama ha solicitado el privilegio con respecto a la vigilancia de la Agencia de Seguridad Nacional, las escuchas telefónicas ilegales y otras actividades (American Academy, 2OI 2: 20).

a) Reflexión del profesor Benkler. Benkler cita los Pentagon Papers: «Este poderoso Ejecutivo tiene la responsabilidad de mantener sus propias operaciones con suficiente seguridad y sabiduría para asegurarse de que sólo lo que necesita clasificar es de hecho clasificado, y lo que se clasifica no consigue ser filtrado». ${ }^{30}$ De esta manera, para él Wikileaks en ningún caso es responsable de garantizar o responder porque los secretos sigan siéndolo.

30. También a propósito de los Papeles del Pentágono hace la distinción de que en ese caso el punto de preocupación era la censura previa, en cambio nuestro objeto de estudio son las posibilidades del eventual juicio y éxito de un procedimiento contra Assange, y se hace una pregunta: ¿creemos que un tribunal que consideró que la Primera Enmienda, que autoriza a los periódicos a publicar, simplemente permitiría al gobierno procesar y encarcelar a los periodistas después de los hechos? Eso sería una burla a la protección e impondría mucho mayor enfriamiento en la publicación que el riesgo de una medida cautelar (Benkler, 20II: 34-35). 
b) Opinión del profesor Schoenfeld (American Academy, 20I 2: 2425). Dice que para mantener la seguridad del país, el gobierno genera una gran cantidad de secretos de muchos tipos. También sostiene que no se pueden dar a conocer los métodos de inteligencia para rastrear ni revelar las vulnerabilidades de los puentes, túneles y edificios.

No obstante, argumenta que de la misma manera está en juego el carácter de la democracia estadounidense. Dice que vivimos en una sociedad abierta en la que el secreto es la antítesis del ideal democrático. El secreto podría ser utilizado para cubrir la corrupción y el delito. Es así como dependemos de la libertad de prensa para que se nos proporcione información acerca de lo que el gobierno está haciendo a nombre de su pueblo, incluyendo algunas de las cosas que están en secreto. De hecho, asegura, gran parte de lo que leemos en los periódicos acerca de los asuntos exteriores se basa en la presentación de informes sobre secretos de Estado. Manifiesta que la publicación periódica de los secretos es parte del sistema estadounidense, y así debe ser.

Siguiendo con su razonamiento, plantea que, así como nosotros queremos una prensa para informar sobre secretos, ésta debe hacerlo bajo el imperio de la ley. Es decir, la prensa — que por cierto, dice, incluye Wikileaks - ha de ser susceptible de acciones de la fiscalía cuando viola las leyes que regulan el secreto. Luego, se hace la siguiente pregunta: ¿la existencia de estas leyes significa que los periodistas deben ser procesados cada vez que publican un secreto de Estado? Y responde: por supuesto que no. Dice que es un hecho reconocido el que el gobierno de Estados Unidos utiliza el sello de secreto promiscuamente. Por lo tanto, el dilema no sería si la prensa siempre debe ser procesada por publicar secretos, sino si la prensa puede ser procesada cuando publica secretos que ponen al país en peligro. Da como ejemplo del daño que se puede causar a un país el ocurrido durante la Primera Guerra Mundial, en la que el Chicago Tribune publicó un artículo sugiriendo que Estados Unidos había descubierto los códigos de la marina japonesa. El gobierno en un principio quiso actuar utilizando la Ley de Espionaje, pero luego decidió retirar los cargos para no darle mayor publicidad al artículo. En 2006, The New York Times reveló el funcionamiento de un programa de lucha contra el terrorismo que seguía el movimiento de fondos de al-Qaeda. El programa era legal y operaba bajo órdenes lícitas. La publicación hizo que una de las principales fuentes sobre al-Qaeda dejara de estar disponible. 
Luego, a propósito de Wikileaks, dice que en nombre de la transparencia se han arrojado a Internet indiscriminadamente miles de documentos secretos, en muchos casos completamente sin editar, aunque muchos de ellos son inocuos.

Algunos, estima, claramente ayudan a entender mejor el papel de Estados Unidos en el mundo, pero otros harían un grave daño. Da el ejemplo de un documento publicado por Wikileaks que describiría los dispositivos de interferencia empleados por los soldados estadounidenses en Irak para codificar las señales utilizadas por los insurgentes para detonar bombas en las carreteras desde una ubicación remota. El documento habría dado información específica sobre cómo funcionaban y qué frecuencias se bloqueaban. Luego se hace la siguiente pregunta: ¿hay alguna persona razonable que crea que debería ser legal publicar las contramedidas secretas que nuestros soldados utilizan para evitar volar por los aires en el campo de batalla? ¿La prensa es libre de revelar la identidad de los agentes de la CIA encubiertos? ¿Quién decide esto? Plantea que lo que el pueblo estadounidense ha decidido se manifiesta a través de las leyes aprobadas por sus representantes electos, y la decisión, según su opinión, es que la publicación de ciertos tipos de secretos es susceptible de órdenes de procesamiento. ${ }^{31}$

Ahora bien, en sentido contrario se manifiesta el profesor Geoffrey Stone, quien no está de acuerdo con el razonamiento de Schoenfeld, ya que estima que no es del todo claro que se apoye castigar las expresiones meramente porque causen daño. Estima que la evaluación del discurso o relato también tiene importancia (Stone, 20I I: I I7-I I8).

\section{¿Se ha expuesto la seguridad nacional de Estados Unidos a un daño grave e irreparable?}

a) Posición del profesor Stone. Stone estima que las razones por las que los funcionarios del gobierno defienden el secreto son muchas y variadas

3I. A lo que Shoenfeld responde que «en cuanto al secreto de seguridad nacional y la cuestión de los daños, vamos a tomar un ejemplo en el que creo que todos podemos estar de acuerdo: que la publicación de las identidades de los agentes encubiertos de la CIA puede causar daño a las personas. En efecto, el Congreso actuó para que sea ilegal publicar los nombres» (American Academy, 20I 2: 24-25). 
y van desde las verdaderamente convincentes hasta las notoriamente ilegítimas. A veces, dice, efectivamente es por temor a que la divulgación de cierta información pueda perjudicar gravemente la seguridad de la nación, como la presentación de planes detallados de batalla en su víspera. Otras veces, es simplemente porque no quieren hacer frente a la crítica pública de sus decisiones e inclusive porque la revelación expondría su propia incompetencia, estupidez o maldad (Stone, 20I I: I07).

Piensa, también, que la contribución de la divulgación al discurso público puede variar ampliamente dependiendo de la naturaleza de la información y las circunstancias, y que la divulgación de cierta información clasificada puede ser extremadamente valiosa para el debate público; por ejemplo, la revelación de programas de gobierno posiblemente ilegales o inconstitucionales, como el uso secreto de interrogatorios coercitivos o la autorización secreta de vigilancia electrónica generalizada. La revelación de otra información confidencial, sin embargo, podría ser de poco o ningún valor para el legítimo debate público; por ejemplo, la publicación de las identidades específicas de los agentes estadounidenses encubiertos en Irán por ninguna otra razón que la exposición (Stone, 20II: IO8).

El verdadero dilema, según Stone, surge cuando la divulgación pública de información secreta es tan perjudicial para la seguridad nacional, como valiosa para el debate público e invita a hacer la siguiente suposición: el gobierno inicia un estudio sobre la eficacia de las medidas de seguridad en las centrales nucleares del país y llega a la conclusión de que varias plantas de energía nuclear son especialmente vulnerables a ataques terroristas. Por un lado, la publicación del informe revelaría vulnerabilidades a los terroristas. Por otro, podría alertar al público sobre la situación para que los ciudadanos puedan presionar a los funcionarios del gobierno para remediar los problemas, y mediante la autonomía de los ciudadanos, soliciten rendir cuenta a los funcionarios públicos que están garantizando su seguridad. ¿El estudio debería mantenerse en secreto o debería ser divulgado al público? En teoría, plantea, habría que establecer si el valor de la exposición a la deliberación pública informada supera su peligro para la seguridad nacional. Pero, luego, dice que sería simplificar demasiado el análisis, siendo extremadamente difícil medir objetiva, coherente y predeciblemente el «valor» de la divulgación o su «peligro» para la seguridad nacional (Stone, 20I I: I08). 
Siguiendo con su argumentación plantea: pensemos primero en personas comunes que no sean empleados públicos. ¿Serán legalmente responsables por revelar información a otro con el propósito de publicarla? ¿Los derechos de la Primera Enmienda de los empleados públicos son diferentes? (Stone, 20I I: I09).

En general, dice, una persona común y corriente tiene un amplio derecho a revelar información a los periodistas u otras personas con fines de publicación. Sin embargo, existen limitaciones. ${ }^{32}$

¿Cuándo podría el gobierno prohibir a un individuo u organización la publicación o difusión de información clasificada filtrada ilegalmente? Dice que en toda la historia de Estados Unidos, el gobierno nunca ha enjuiciado con éxito a nadie (que no sea un empleado del gobierno) para impedir la difusión pública de dicha información (Stone, 20II: II3).

Describe que debido a que nunca ha existido un procesamiento exitoso, el Tribunal Supremo nunca ha tenido ocasión de pronunciarse sobre este tipo de casos. Lo más cerca que ha llegado a tal situación fue en los Pentagon Papers, en el que el Tribunal de Justicia declaró inconstitucional el esfuerzo del gobierno para prohibir la publicación. La opinión del juez Stewart capta la opinión de la Corte: «Se nos pide», consideró, «impedir la publicación de material que el Ejecutivo insiste en que no debería hacerse, en el interés nacional. Estoy convencido que el Ejecutivo tiene razón con respecto a algunos de los documentos en cuestión. Pero no puedo decir que la divulgación de cualquiera de ellos seguramente

32. «En primer lugar, el Tribunal Supremo ha reconocido desde hace tiempo que hay ciertas 'clases limitadas de expresión', tales como las declaraciones falsas de hechos, obscenidades y amenazas, que 'no son parte esencial de cualquier exposición de ideas' y, por tanto, tal expresión puede restringirse sin satisfacer las exigencias habituales de la Primera Enmienda. En segundo lugar, los particulares pueden contraer voluntariamente con otros particulares la obligación de limitar su discurso. La violación de dicho acuerdo privado puede ser punible como un incumplimiento de contrato. En tercer lugar, puede haber situaciones, de todas formas excepcionales, en la que una persona da a conocer previamente la información no pública a un periodista en circunstancias en que la publicación sería tan peligrosa para la sociedad que el individuo puede ser castigado por revelar la información al periodista con fines de mayor difusión. En general, sin embargo, la Primera Enmienda garantiza a los individuos una amplia libertad para compartir información con otras personas con fines de publicación»(Stone, 20II: IO9-IIO). 
resultará en un daño directo, inmediato e irreparable a nuestra nación o a su gente». ${ }^{33}$

Así, en el caso de los Papeles del Pentágono, la Corte sostuvo que aunque los funcionarios electos tienen amplia autoridad para mantener la información en secreto clasificado, una vez que la información llega a otras manos el gobierno sólo tiene autoridad limitada para evitar su ulterior difusión. ${ }^{34}$

Finalmente, subraya que el juez Stewart observó en el caso de los Papeles del Pentágono que, a pesar de que la publicación de algunos de los materiales podrían dañar «el interés nacional», su difusión no podía constitucionalmente prohibirse salvo que su divulgación implicase «sin duda como resultado directo, inmediato e irreparable daño a nuestra nación o a su gente». Así, plantea que, en primer lugar, el mero hecho de que la difusión pueda perjudicar el interés nacional no significa que el daño sea mayor que los beneficios de la publicación; en segundo lugar, un "caso a caso" de equilibrio de daño versus beneficio, resulta difícil de manejar, es impredecible e impracticable; en tercer lugar, dice que tal como hemos aprendido de nuestra propia historia, hay grandes presiones que conducen tanto a los funcionarios del gobierno como al propio público a subestimar los beneficios de la publicación y exagerar su daño potencial en momentos de angustia nacional. Una norma estricta de peligro claro y presente, dice, serviría como una barrera para protegernos

33. Stone (20I I: I I3-I I4) citando el juicio The New York Times Co. vs. United States, 403 U.S. 7I 3 (197I).

34. «Esto puede parecer torpe, incluso incoherente: si el gobierno puede prohibir constitucionalmente a los empleados públicos la revelación de información clasificada a los demás, ¿:por qué no puede imponérselo a los destinatarios? Se podría dar vuelta con la misma facilidad la pregunta: si los individuos tienen un derecho a publicar información clasificada a menos que de la publicación 'sin duda resultará un daño directo, inmediato e irreparable a nuestra nación y su gente’, ¿por qué se le debe permitir al gobierno prohibir a sus empleados revelar dicha información a los demás simplemente porque representa un peligro potencial para la seguridad nacional? Si vemos el tema desde la perspectiva de cualquiera de los intereses del público en el discurso informado o el interés del gobierno en el secreto, parece que la misma regla lógica debería aplicarse tanto a los empleados públicos como a los que se les difundirá la información. Las grandes diferencias entre las normas que rigen a los empleados públicos, por una parte, y a otros oradores, por el otro, representa un misterio» (Stone, 20I I: I I4). 
contra este peligro; y en cuarto lugar, un principio central de la Primera Enmienda es que la supresión vía intervención pública debe ser el último recurso del gobierno para hacer frente a un problema potencial. Si hay otros medios por los cuales el gobierno puede prevenir o reducir el riesgo, se deben agotar otros medios antes de que se pueda suprimir la libertad de expresión. Esto, también, es una premisa esencial de la norma de peligro claro y presente. En la hipótesis de la clandestinidad, la manera más obvia en que el gobierno puede evitar el peligro es garantizando seriamente que la información perjudicial, en primer lugar, no se filtrará (Stone, 2OII: II4-II5).

La solución para él es intentar conciliar los valores del secreto y de la rendición de cuentas, garantizando tanto una fuerte autoridad para prohibir fugas al gobierno como un derecho amplio de otros para difundirlos (Stone, 20II: II6).

Tres preguntas permanecen. Primero, ¿la misma norma constitucional regularía las restricciones previas y los procesos penales? Segundo, ¿qué tipo de revelaciones podrían satisfacer el estándar de peligro claro y presente? Y tercero, ¿cómo debemos tratar la información que satisface tanto el estándar de peligro claro y presente como el de contribuir significativamente al debate público? (Stone, 20II: I I6).

Para responder la primera pregunta expone que en el caso de los $\mathrm{Pa}$ peles del Pentágono, la Corte hizo hincapié en que se trataba de un mandamiento judicial contra el discurso. Esta orden judicial habría sido la de una restricción previa, un tipo de restricción al discurso que, en palabras de la Corte, tiene una particularmente «fuerte presunción contra su constitucionalidad». Plantea que, en relación con la expresión, en el corazón mismo de la Primera Enmienda —el discurso sobre la conducta misma del gobierno- la distinción entre la restricción previa y el procesamiento criminal no debería ser de mucho peso. El criterio aplicado en los Pentagon Papers es esencialmente el mismo estándar que sería aplicable en un proceso penal de una organización o individuo que difunda públicamente información sobre la conducta del gobierno. El estándar de peligro claro y presente, estima, no se ha limitado a los casos de censura previa (Stone, 20II: II6).

Para la segunda, los ejemplos ofrecidos tradicionalmente eran «las fechas de salida de los transportes» o la «ubicación de las tropas de combate», precisamente en tiempos de guerra. La publicación de esa 
información en ese instante hace que las tropas estadounidenses se vuelvan vulnerables a un ataque enemigo y frustre los planes de batalla ya en marcha. Otros ejemplos podrían incluir la publicación de la identidad de los agentes encubiertos de la CIA o la revelación pública de que el gobierno ha violado el código secreto de al-Qaeda, alertando así al enemigo a cambiar su sistema de cifrado. En situaciones como éstas, el daño de la publicación podría ser lo suficientemente probable, inminente y grave para justificar el castigo de la divulgación, estima (Stone, 20I I: I I6).

Para la tercera, dice, una característica importante de estos ejemplos a menudo pasa desapercibida. Lo que hace convincentes a estas situaciones no es sólo la posibilidad inminente y la magnitud de los daños, sino también el supuesto implícito de que este tipo de información no contribuye significativamente al debate público. En la mayoría de los casos, no hay una necesidad evidente de que el público conozca el secreto de las «fechas de salida de los transportes» o el secreto de la «ubicación de las tropas estadounidenses» en la víspera de la batalla. En ese instante estas cuestiones son irrelevantes para la discusión política. Después del hecho, argumenta, por supuesto que esa información puede ser crítica para la evaluación de la efectividad de nuestros líderes militares, pero en el mismo momento en que los barcos se ponen a navegar o que las tropas se ponen a atacar, no está tan claro lo que aporte esa información al debate público. Su punto, expresa, no es que estos ejemplos impliquen expresiones de «bajo» valor en el sentido convencional del término, sino que implican que la información no parece especialmente «de interés periodístico o noticioso» en el momento de la publicación (Stone, 20 I I: I I7).

Sugiere suponer, por ejemplo, que un periódico informa con exactitud que las tropas estadounidenses en Afganistán asesinan recientemente a veinte miembros de al-Qaeda a sangre fría. Como resultado de esta publicación, al-Qaeda secuestra y asesina veinte ciudadanos estadounidenses. ¿Puede el periódico constitucionalmente ser castigado por la divulgación de la masacre inicial? La respuesta, piensa, debe ser no. Incluso, dice, existiendo un peligro claro y presente de que una represalia pueda sobrevenir, e incluso si estamos de acuerdo en que se trataría de un grave daño, la información es demasiado importante para el pueblo estadounidense como para castigar su divulgación (Stone, 20II: I I7).

Lo que propone el profesor Stone es que para justificar la sanción penal de la prensa por la publicación de información clasificada, el gobier- 
no debe demostrar no sólo que el demandado publicó información clasificada, cuya publicación podría resultar en un daño probable, inminente y grave para la seguridad nacional, sino que también deberá demostrar que la publicación no contribuiría significativamente al debate público (Stone, 20II: II7-II 8).

b) Posición del profesor Benkler. A propósito del peligro claro y presente que tendría que demostrarse para restringir la expresión en interés de la seguridad nacional, Benkler plantea que la larga historia desde el caso Schenck v. United States (I919), Whitney v. California (1927) y Brandenburg $v$. Ohio (I969), para abrazar el estándar de "peligro claro y presente», terminó requiriendo una combinación similar de alta probabilidad, de alto daño y de la inmediatez del proceso. Cuando la Corte Suprema se puso en el contexto de considerar la responsabilidad penal de un locutor que había transmitido materiales ilegales, estimó que la Primera Enmienda no permite el enjuiciamiento de un periodista que transmite información veraz de interés público «en ausencia de una necesidad de primer orden». ${ }^{35}$ Por lo tanto, el estándar para la censura previa y el de la persecución penal por la publicación de materiales veraces de interés público parece ser básicamente el mismo y sumamente estricto (Benkler, 20I I: 35). ${ }^{36}$

c) Posición del abogado Elsea del CRS. Elsea expone que cuando se trata de limitar el habla en función de su contenido, la Corte Suprema aplica en general un «estricto escrutinio", lo que significa que va a defender una restricción basada en el contenido sólo si es necesario para «promover un interés apremiante», y es «el medio menos restrictivo para promover el interés articulado». ${ }^{37}$ La protección de la seguridad nacional ante amenazas externas es, sin duda, un interés gubernamental

35. Benkler (20I I: 35 ) citando Bartnicki vs. Vopper 532 U.S. 5I4, 528 (200I) (citando a su vez a Smith vs. Daily Mail Publishing Co., 443 U.S. 97, I03 (I979)).

36. «En el trasfondo de esta barrera extremadamente alta para ambos, censura previa y enjuiciamiento criminal, tal vez no sea sorprendente que los esfuerzos de la Administración Bush para enjuiciar al The New York Times por sus revelaciones sobre el programa de la Agencia de Seguridad Nacional de espionaje interno, y al Washington Post por la presentación de informes sobre la existencia de sitios operados por la CIA en Europa del Este, fueron abandonados» (Benkler, 20I I: 35 ).

37. Elsea (20I3: 2I) citando a Sable Communications of California v. Federal Communications Commission, 492 U.S. I I 5, I 26 (I989). 
apremiante (Elsea, 20I3: 2I). Durante mucho tiempo se ha aceptado que el gobierno tendría una necesidad imperiosa de suprimir ciertos tipos de discurso, sobre todo en tiempos de guerra o de riesgo elevado de hostilidades. ${ }^{38}$ Discursos susceptibles de incitar a la violencia inmediata, por ejemplo, también pueden ser suprimidos. ${ }^{39} \mathrm{El}$ discurso que daría ventaja militar al enemigo extranjero también sería susceptible de regulación gubernamental. ${ }^{40}$

Se pregunta: ¿qué derechos de la Primera Enmienda están implicados? Argumenta que si el gobierno tiene una necesidad imperiosa de sancionar la divulgación de información clasificada, se activa si la divulgación tiene el potencial de causar daños a la defensa nacional o a las relaciones exteriores de los Estados Unidos. Los daños reales, dice, no necesitarían ser probados, pero el daño potencial debe ser más que meramente especulativo e incidental. Por otra parte, la Corte ha dicho que: «la acción del Estado para castigar la publicación de información veraz rara vez cumple las normas constitucionales». ${ }^{41} \mathrm{Y}$ se ha descrito el propósito detrás de la garantía constitucional de la libertad de prensa como la protección de «la libre discusión de los asuntos gubernamentales». ${ }^{42}$

Aunque la información, debidamente clasificada, de ser revelada a una persona no autorizada podría potencialmente causar daño a la seguridad nacional de los Estados Unidos, ${ }^{43}$ no se desprende necesariamente que

38. Elsea (2013: 22) citando Schenck v. United States, 249 U.S. 47 (I9I9) (formulando el test de «peligro claro y presente»).

39. Elsea (2013: 22) citando Brandenburg v. Ohio, 395 U.S. 444 (I969).

40. Elsea (20I3: 22) citando a su vez Near v. Minnesota, 283 U.S. 697, 7I6 (I93I). «Nadie duda de que un gobierno puede evitar la obstrucción real a su servicio de reclutamiento o de la publicación de las fechas de salida de los transportes o el número y la ubicación de las tropas».

4I. Elsea (2013: 22) citando a su vez Bartnicki vs. Vopper, 532 U.S. 5I4, 527 (200I) (citando a Smith v. Daily Mail Publishing Co., 443 U.S. 97 (I979)).

42. Elsea (2013: 22) citando a su vez Mills v. Alabama, 384 U.S. 214, 2 I 8 (I966).

43. Elsea (2013: 22) citando a su vez Exec. Order No. I3526, 75 Fed. Reg. 707 \I.2 (January 5, 2010) ("Classified National Security Information»). La sección I.3 define tres niveles de clasificación: I) «Top Secret» se aplicará a la información de la que podría esperarse razonablemente que su divulgación no autorizada pueda causar daño excepcionalmente grave a la seguridad nacional, que la autoridad de clasificación original es capaz de identificar o describir. 2) «Secreto» se aplicará a la información de la que podría esperarse razonablemente que su divulgación no autorizada pueda causar un perjuicio 
la clasificación del gobierno por sí sola sea determinante de la cuestión en el contexto de un proceso penal. Los tribunales han adoptado como elemento de los estatutos de espionaje el requisito de que la información en cuestión debe ser «mantenida estrechamente». ${ }^{44} \mathrm{Si}$ bien la clasificación del gobierno sería una fuerte evidencia para apoyar esta afirmación, los jueces han reconocido que el gobierno debe demostrar que la divulgación de información específica de la defensa nacional tenga el potencial de dañar los intereses estadounidenses, pues de otra manera la Ley de Espionaje sería un medio para castigar a los denunciantes que revelen información embarazosa para los funcionarios públicos más que para castigar la puesta en peligro de la seguridad nacional (Elsea, 2013: 23).

\section{CONCLUSIONES}

Sostenemos que la Computer Fraud and Abuse Act es inaplicable a Julian Assange, dado que no ha existido acceso no autorizado a computadores o redes de computadores del gobierno estadounidense. Tampoco podría hablarse de complicidad o encubrimiento, dado que ni siquiera Wikileaks podía acceder a la identidad de sus fuentes en su sistema ideado para protegerlas.

Estimamos que la Ley de Espionaje tampoco es aplicable a las conductas de Wikileaks. Pensamos que al recibirse u obtenerse la información no se tuvo intención de lesionar a Estados Unidos ni que la obtención de ella fuese motivada para provocarle daño; tampoco, que al poseerse sin autorización documentos de la defensa de Estados Unidos y comunicarlos voluntariamente a quien no tenía derecho a recibirlos se tuvo la intención de dañarlo. Estimamos que no se podrá probar ni la intención específica de daño ni el daño potencial a la seguridad nacional.

En lo referido a la prueba de la intención específica, fue la decisión en Hartzel v. Estados Unidos (I944) la que esbozó el criterio estándar de un

grave a la seguridad nacional, que la autoridad de clasificación original es capaz de identificar o describir. 3) «Confidencial» se aplicará a la información de la que podría esperarse razonablemente que la divulgación no autorizada pueda causar daño a la seguridad nacional que la autoridad de clasificación original es capaz de identificar o describir.

44. Elsea (20I3: 22) citando a su vez United States v. Heine, I5I F.2d 8I3 (2d Cir.I945). La información debe ser «estrechamente sostenida» para considerarse «en relación con la defensa nacional» en el sentido de las leyes de espionaje. 
procesamiento exitoso. ${ }^{45}$ Ello fue reforzado por el juez del caso AIPAC, al establecer que un enjuiciamiento exitoso debe probar que el acusado sabe que la divulgación de la información de defensa es dañina para Estados Unidos y lo hace con intención de que ese daño se materialice. No se podría probar, porque al recibirse u obtenerse los documentos no se sabía qué se obtenía ni desde dónde, porque su propio sistema de protección de fuentes hacía que no tuviese acceso a los datos de proveniencia de los documentos, siendo la intención aplicable en este contexto la intención genérica del sitio Wikileaks, la de recibir documentos en que se contenga información de posibles malas prácticas de gobiernos o de empresas, no la de hacer daño a los Estados Unidos.

Tampoco podrá probarse o se hace aun más complejo probar la intención dañosa en la segunda hipótesis en relación a la posesión y comunicación del material, pues debemos hacer mención a las solicitudes de Julian Assange -incluso desde los diarios de guerra de Afganistán — al gobierno norteamericano a través del The New York Times, requiriendo información acerca del estándar en que una publicación podría constituir una amenaza para las personas, las cuales no fueron respondidas en abstracto, pero tampoco en concreto, existiendo esta posibilidad, pues The New York Times enviaba a su gobierno cierta cantidad de documentos a modo de alerta temprana antes de cada publicación. Por lo tanto había, por una parte, una intención de prevenir cualquier tipo de daño, y, por otra, un conocimiento previo de lo que se publicaría por parte del eventual afectado. ${ }^{46}$

45. «Salvo que se encuentren pruebas suficientes de que un jurado pudiese inferir más allá de una duda razonable de que tenía la intención de llevar a cabo las consecuencias específicas prohibidas por la ley [...] la libertad de expresión y de prensa están protegidas».

46. A ello hay que agregar que en el fallo sobre el caso Papeles del Pentágono un juez de la mayoría declaró que la expresión «comunica» de la ley no puede identificarse con la expresión "publica», por lo que la publicación de la información no sería una conducta reprochada. Además, a mayor abundamiento y en concordancia con la doctrina de la Primera Enmienda expuesta durante el transcurso de la tesis base de este trabajo, resultaría, por decir lo menos, forzado argumentar que el pueblo estadounidense no tuviese derecho a recibir la información, sobre todo de actuaciones ilícitas de quienes los representan y deciden el destino de la nación, como crímenes de guerra, violaciones a los derechos humanos y espionaje internacional. 
Debemos también destacar que la única ocasión en que se invocó directamente contra la prensa la Ley de Espionaje no fue en un proceso penal, sino que en los Pentagon Papers y estamos de acuerdo con el profesor Barron en que al establecerse sus disposiciones desde un lenguaje vago y demasiado amplio se podrían plantear cuestiones de constitucionalidad de la ley.

Finalmente es de interés destacar la opinión de Jones y Ward en relación a que si hubiese sido un mass media el acusado, respondería alegando que su intención era la de informar sobre asuntos pertinentes al self-government, que no se violó la ley ni se dañó al gobierno y así se ha interpretado la intención por los tribunales en circunstancias análogas. Es interesante porque entrega un argumento a la defensa ya que vimos que la garantía a la libertad de expresión y de prensa no es excluyente ni otorga un estatuto especial a la prensa tradicional.

Por los mismos motivos, estimamos que el hecho de que Wikileaks sea o no parte de la prensa no es relevante para solicitar la protección de la Primera Enmienda. Concordamos con el profesor Benkler en el sentido de que la protección de la Primera Enmienda más que al aparato formal que realiza la publicación está dirigida a tutelar el punto de si de lo que trata la información resulta relevante para el debate de la comunidad nacional representada, dada la configuración de realidad necesaria para tomar decisiones colectivas para validar o sustituir a sus propios representantes y en términos más amplios incluso validando y profundizando la misma democracia.

A mayor abundamiento, pensamos que el descontrolado aumento, sobre todo en los últimos años, de la información catalogada como clasificada, desnaturaliza la relación que se supone existente entre su revelación no autorizada y un daño a la seguridad nacional. De hecho, incluso luego de una de las filtraciones de Wikileaks el mismo gobierno de Estados Unidos recalificó el material disminuyendo su calificación, su protección y en definitiva su relación con un daño a la seguridad nacional. Establecido ello por la doctrina, pierde poder el argumento relativo a que una información clasificada revelada produzca per se daño o puesta en peligro de la seguridad de los Estados Unidos.

De esta manera, tendríamos que recurrir a la doctrina del peligro claro y presente. Estimamos que de aplicarse aquella doctrina al caso Wikileaks en el momento de las revelaciones, en el momento presente y 
aun más, en el futuro, resulta cada vez más evidente que no hubo peligro claro y presente para la seguridad nacional estadounidense, pues de los antecedentes conocidos queda claramente establecido que no se ha podido demostrar que una sola persona ligada a sus fuerzas de seguridad nacional haya muerto debido a las filtraciones y que incluso habiendo ocurrido ello tampoco implicaría un riesgo de la entidad requerida por la doctrina para entender que Estados Unidos se encontró en peligro.

Queda cada vez más claro que la información revelada ha sido un aporte para el debate público estadounidense, promoviendo el ejercicio legítimo de la libertad de expresión y de prensa, y que las autoridades lo que realmente han buscado, utilizando el argumento de la defensa del secreto para la seguridad de una nación, es esconder delitos y crímenes secretamente documentados.

\section{REFERENCIAS}

Domscheit-Berg, Daniel (2ori). Dentro de Wikileaks. Mi etapa en la web más peligrosa del mundo. Traducción de Ana Duque de Vega y Carles Anfreu Saburit. Barcelona: Roca.

LeIgh, David y Luke Harding (2OII). Wikileaks y Assange. Un relato trepidante sobre cómo se fraguó la mayor filtración de la historia. Traducción de Mar Vidal e Isabel Merino. Barcelona: Deusto.

O'Hagan, Andrew (2012). Julian Assange. La verdad amordazada. Autobiografía no autorizada. Traducción de Enrique Murillo. Santiago: Lince-Catalonia.

American Academy of Arts \& Sciences (2012). "WikiLeaks \& the First Amendment», Bulletin of the American Academy of Arts \& Sciences, Northwestern University School of Law, Thorne Auditorium, Chicago, IL November I2, 201 I.

Barron, Jerome A. (20II). "The Pentagon Papers case and the WikiLeaks controversy: National security and the First Amendment». Wake Forest Journal of Law \& Policy, I (I): 46-72. Disponible en $<$ http://papers.ssrn.com/sol3/papers.cfm?abstract_id=I8 I 4 I 8 I >.

BenKLer, Yochai (20II). "A free irresponsible press: Wikileaks and the battle over the soul of the networked fourth estate». Harvard Civil Rights-Civil Liberties Law Review, 46: 3 I I-397. Disponible previo a la publicación en <http://benkler.org/Benkler_Wikileaks_current.pdf>. 
Clough, Jonathan (20II). «Data theft? Cybercrime and the increasing criminalization of acces to data». Criminal Law Forum, 22: I45-I70.

ELSEA, Jennifer K. (20I3). "Criminal prohibitions on the publication of classified defense information». Congressional Research Service. Disponible en <http://fas.org/sgp/crs/secrecy/R4I404.pdf>.

Jones, Shaina y Jay Ward Brown (20II). "The Assange Effect: WikiLeaks, the Espionage Act and the Fourth Estate», Media Law Resource Center Bulletin, I I 5-I 47. Disponible en <http://www.lskslaw.com/ documents/WikiArticle(004450I3).pdf>.

Stone, Geoffrey (20I I). "WikiLeaks, the Proposed Shield Act, and the First Amendment». Journal of National Security Law \& Policy, 5: I05-I I 8. Disponible en <http://www.goodtimesweb.org/documentation/o2_Stone.pdf>.

Mcquade, Samuel (2009). Encyclopedia of cybercrime. Westport, Connecticut, Londres: Greenwood Publishing Group.

Cebrián, Mariano (2008). «La Web 2.0 como red social de comunicación e información». Estudios sobre el Mensaje Periodístico, I4: 345-36r. Disponible en <http://revistas.ucm.es/index.php/esmp/article/view/esmpo808 I I0345a>.

\section{SOBRE EL AUTOR}

Felipe Campos Arlegui es Licenciado en Ciencias Jurídicas y Sociales por la Universidad de Chile. Su correo electrónico es < felipe.campos. arlegui@gmail.com>. Este artículo está basado parcialmente en su memoria para optar al grado de Licenciado en Ciencias Jurídicas y Sociales por la Universidad de Chile.

Este trabajo fue recibido el 22 de abril de 2014 y aprobado el 25 de junio de 20 I4. 